\title{
Pump Outs, General and \\ D0 Considerations
}

\author{
G. T. Mulholland \\ December 25,1990
}

\author{
3740.214-EN-274 \\ Revision A \\ January 1, 1991
}




\section{Seal Pump Outs, General and D0 Considerations}

\section{General}

Seal Pump Outs (PO) are provided by specifically designing and providing redundant, or double, seals to create an annular volume that can be "(vacuum) pumped out" to test the integrity of both seals. The value of the technique is most readily apparent in the construction of large piping systems or vessels whose closure is on a different schedule than the nozzle closures, or whose nozzles are serially closed. In the case of D0, for instance, the high voltage boxes were put in place and leak checked before the vessel was closed and independent of the other nozzles. PO use is by no means limited to cryogenics and the supporting vacuum systems, but the discussion here will be limited to cryogenic applications.

POs come in two generic service types; installation, and installation and monitor. The above high voltage box is an example of a static installation service application. Once the item is installed the PO can be, and almost universally is, capped, and revisited only on disassembly and reassembly. POs are constantly monitored after installation only when their seals, through cooldown gradient induced motion, vibration, cyclic load bolt seating, or other dynamic phenomena may degrade in performance over time.

PO seals come in two general temperature service types; ambient and cryogenic temperatures. "O" rings are the predominant warm seal. " $\mathrm{C}$ ", and other (Conoseal, Conflat, soft copper, etc.) metal seals (of copper, Inconel, indium, lead, stainless steel, alone or in combination) make up the usual field of cold seals. The much smaller class of cold seals made of metal spring activated Teflon or other spring activated seal materials have not been used here and will not be further addressed.

In cryogenic work there are four regions that can be interfaced; cryogenic (cold), vacuum, atmosphere, and warm to cold transition nozzles (nozzle). Taken two at a time the four provide 10 possible interfaces. Table 
1. lists the typical applications when $P O$ are used, as practiced and known to the author. The greatest numbers of nozzle seal applications are standard bayonets, but with only one (warm) seal they are excluded from consideration in Table 1.

\section{Table 1. PO Applications}

\begin{tabular}{|c|c|c|c|c|c|}
\hline PO & At & Install $L$ & Warm & Cold & \\
\hline Interface & Install & Monitor & Seal & Seal & Remarks \\
\hline 1.Cold-Cold & $\mathbf{X}$ & & & $\mathbf{X}$ & Rare \\
\hline 2. Cold-Vac. & $\mathrm{X}$ & $\mathbf{X}$ & & $\mathbf{X}$ & Common, Note 1 . \\
\hline 3. Cold-Atmos. & $\mathbf{X}$ & & & $\mathrm{X}$ & Rare \\
\hline 4. Cold-Nozzle & $\mathrm{X}$ & $\mathbf{X}$ & & $\mathbf{X}$ & Rare, similar to Cold-Vac. \\
\hline 5. Vac.-Vac. & $\mathrm{X}$ & $\mathbf{X}$ & $\mathbf{X}$ & opt & Rare, see Note 3 \\
\hline 6. Vac.-Atmos. & $\mathbf{X}$ & rare & $\mathbf{X}$ & & Common \\
\hline 7. Vac.-Nozzle & $\mathrm{X}$ & rare & $\mathbf{X}$ & opt. & Assumes warm end \\
\hline $\begin{array}{l}\text { 8. Atmos.-Nozzle } \\
\text { 9. Atmos.-Atmos. } \\
\text { 10. Nozzle-Nozzle }\end{array}$ & $X$ & rare & $\mathrm{X}$ & opt. & $\begin{array}{l}\text { Common, Note } 2 . \\
\text { None known } \\
\text { None known }\end{array}$ \\
\hline
\end{tabular}

\section{Notes;}

1. Vacuum means insulating vacuum.

2. Nozzles come in a wide variety of applications and complications, see discussion.

3. The second Vacuum is an independent (better) vacuum.

\section{Specific Application Discussion, General}

The case for installation (assembly) POs is obvious on large systems, especially those with complicated serial assembly, and requires no further comment. It is, however, useful to ask why vacuum jacketed piping systems are assembled with one seal. At installation, VJ lines can be isolated, the small volumes are quickly vacuum pumped and purged, easily disassembled for the repair or replacement of seals, and the single seal fabrication cost is lower. They do represent a large, successful, unmonitored, part of all such operating seals. In the Table 1. classification they would be an Atmos.-Nozzle application.

The Install/Monitor column of table 1 . requires comment. It is common for those unable to avoid large vessel cold seals in an insulating vacuum, to monitor the $P O$ as a diagnostic for the insulating vacuum. Bubble chambers with flanges as large as 60" diameter with 200 psi 
cryogens on one side and $10^{* *-6} \mathrm{mmHg}$ on the other, are the best (ColdVac.) example. Note too, that bubble chambers typically have inflatable seals that can be further pressurized during operation. Accelerators seem to make Cold-Vac. connections below ca. 6" diameter w/o double seals and POs, there are just two many to handle. The typical accelerator approach is to optimize the design to increase the ease of assembly, make-up, and long term reliability. The Tevatron patterns itself after a large, segmented, piping system; liquid volume is ca. $10,000 \mathrm{gls}$ total in 24 sections. The typical commissioning unit is a section.

Vac.-Vac., Vac.-Atmos., Vac.-Nozzle, and Atmos.-Nozzle applications have been PO monitored in, respectively, 1) bubble chamber insulating-towindow vacuum interfaces, 2) on large, e.g. 22' diameter vacuum sphere flanges, at least initially, 3) and 4) bubble chamber window vacuum installations. All monitoring was done for reasons connected with the shock and vibration endemic to bubble chambers.

Cold seals are typically applied when any viable failure can make the seal area cold and lead to a escalation of the event; where cold takes a definition of $-40 \mathrm{C}$ limit for warm, typically " $\mathrm{O}$ " ring, seals. There is plenty of evidence for avoiding the use of " $O$ " rings at, or approaching, that temperature. Note that the inside-outside order of the warm and cold seals is arbitrary, except that the cold seals should be on the warm side for large leaks in PO monitored applications.

\section{Specific Application Discussion, D0}

D0 has three cryostats, and each has 28 pressure vessel (Atmos.Nozzle) POs with 112 seals and 5 (Vac.-Atmos.) vacuum vessel POs with 10 seals, see the list in Appendix A. The CC Cryostat is the basis for the following discussion, although the generalities apply to all three cryostats.

Of the 112 PV seals, 100 are of the submerged nozzle, Atmos.-Nozzle, variety, i.e. the nozzle lower opening is significantly below the operating liquid level. The equilibrium liquid level in these nozzles is maintained by the gas tightness of the nozzle and the normal heat leak. Note that if the POs are continuously monitored (constantly vacuum pumped) any air leak that might develop from the atmosphere would have its inward flow enhanced by the vacuum pumping, and the flow due to a PV outward leak would be similarly enhanced and makes the seal region colder. The application of the PO monitor to either leak worsens the situation, as contrasted to a Cold-Vac. PO whose use may both help diagnose and 
improve the insulating vacuum.

If there is a significant concern to monitor for the failure of signal board seals, the temperature of the top flange could be measured by an RTD, and not contribute to the air infiltration, cold flow, equipment failure (the dedicated PO vacuum pump and auxiliaries), or the human error possibilities of the PO. Note that the close proximity of the seals can couple the temperature effects of one to the others, and only warm seals ${ }^{1}$ have been provided here.

It has been postulated that the PO lines could be used to deliver warm GAr to keep, especially, the signal board seal area warm in the event of a leak. Note that 1) there is no way to currently measure the temperature of the seal area, 2) when the inner seal leaks, turning off the PO monitor would warm the area, and 3 ) there is no way to know how to set a warming flow into the cryostat. Note that, aside from other effects, the refrigeration load of such a warming flow can be $265 \mathrm{~W} / \mathrm{cfm}$. If warming the flange is deemed important, a DC heater and RTD could be fitted to the flange. That would allow the controlled application of heat, the ability to measure the result, but have no real or potential negative side effects. That suggestion was made some time ago.

It has been suggested that the PO monitor flow be gas analyzed to determine the fraction of air in the flow, and as a measure of the function of the outer seals. The outer seals have been tested to be helium leak tight, and the inner seals are known to have serious reservations at the level of ca. $10 \%$ of the total number. Any measurement under those conditions would find only GAr. It is hard to imagine how that situation might significantly change unless the seals are physically damaged or they get cold. Continued operation of the detector would almost certainly be guided by criteria other than a minor leak, in any case.

The clear conclusion is to install two seals that are tight wherever possible ${ }^{2}$. If there are finite, irreparable, inner seal leaks, but no outer seal leaks, the PO should be capped (zero pumping). The PO has served the installation function and provided one seal w/o reservations (remember the standard bayonets). Capping avoids all the negatives of pumping and flowing, and guards against the equipment or human error that might lead to the escalation of a leak, contamination, or both. There is no reason to believe, except for temperature inducements, the seals will degrade if left

\footnotetext{
A special endorsement has been provided.

${ }^{2}$ An installation activated seal could better address the difficult tolerance problems with the signal board radial seals.
} 
undisturbed. The site is full of single "O" ring, low heat leak, LN2 bayonets that work year in and year out, even while out in the elements.

The safety argument has been made ${ }^{3}$ that there is no serious concern (nozzle liquid level changes) for the outer seals getting cold; their absence doesn't create a leak larger than the required event threshold in the current, tight tolerance, fit of boards to the box. That is, it is intrinsically safe. That is consistent with a capped PO. The situation of a leaking inner seal(s) and a monitored PO provides another flow path, and was not considered.

Lastly, note that so long as there is access, it is possible to make the $1 / 4$ " connections to those POs at any time. The opposite view has been put forward.

POs are intended to aid installation and can be used to monitor initially tight seal installations. They do not substitute for improperly installed seals, and as monitors can, in fact, make a leaking seal installation worse. Other monitoring means can be applied where the need exists.

\footnotetext{
${ }^{3}$ J. Sculli at a D0 Cryosafety meeting
} 
Appendix A.

\section{Cryostat Pump Outs}

$\underline{\text { Item Each Cryostat }}^{\mathrm{b}} \quad \underline{\text { PO }}$ Seals $\underline{\text { Remarks }}^{\mathrm{a}}$

1. Signal Box Flange 4(1) 8 O,C

2. Signal Box Plates 4(2) 16 O,I

3. Signal Boards (X8) 4(1) 64 O,O

4. HV Box Flange 2(1) 4 O,C

5. HV Box Plates 2(1) 4 O,I

6. Instr. Box Flange 1(1) 2 O,C

7. Instr. Box Plates 1(1) 2 O,I

8. Relief Valve 2(1) 4 O,I

9. Relief Valve Gate 2(1) 4 O,I

10. Rupture Disk 2(1) 4 O,I

11. other

Total

$\frac{-}{28} \frac{}{112}$

Notes;

a). $\mathrm{O}=$ "O" ring, $\mathrm{I}=$ Indium, $\mathrm{C}=$ "C" seal

b). Vacuum PO's each cryostat; DP, DP spool, rough pump, cold valve access, and vacuum relief ports. Totals, PO; 5 , seals; 10 